\title{
Principal Curves to Extract Vessels in 3D Angiograms
}

\author{
Wilbur C. K. Wong \\ cswilburecse.ust.hk \\ Albert C. S. Chung \\ achung@cse.ust.hk \\ Lo Kwee-Seong Medical Image Analysis Laboratory \\ Department of Computer Science and Engineering \\ The Hong Kong University of Science and Technology \\ Clear Water Bay, Hong Kong
}

\begin{abstract}
Segmentation of blood vessels and extraction of their centerlines in $3 D$ angiography are essential to diagnosis and prognosis of vascular diseases, and advanced image processing and analysis. In this paper, we propose a semiautomatic method to perform those two tasks simultaneously. A user supplies two end points to the algorithm and a vessel centerline between the two given points is extracted automatically. Local vessel widths are estimated as byproducts. Additional anchor points can be added in between to handle difficult situation. Our method is based upon a polygonal line algorithm. This algorithm is used to find principal curves, nonlinear generalization of principal components, from point clouds. We discuss an application of principal curve to vessel extraction from a theoretical view point. A novel algorithm is then proposed for the application. No data interpolation is needed in the algorithm and centerlines extracted are adaptive to the vasculature complexity on account of their nonparametric representation. We have tested the method on two synthetic data sets and two clinical data sets. Results show that it has high robustness to variation in image resolution, voxel anisotropy and noise. Moreover, centerlines obtained are in subvoxel precision and local widths estimated are accurate under limit of image resolution.
\end{abstract}

\section{Introduction}

Three-dimensional (3D) angiograms very often make diagnosis and prognosis of vascular diseases integral. They provide comprehensive information on a circulation of interest. Segmentation of these images, therefore, has its merits in a clinical environment. It offers physicians to access patient-specific 3D vascular models for effective and efficient data comprehension. Centerline (axis) of vessels is as important as the segmentation. It is a piece of crucial information in advanced image analysis, viz. generating fly-throughs of virtual endoscopy [8], studying populations' vessel attributes [3] and making real-time 3D-2D vascular registration possible [7].

Algorithms to extract vessel centerlines can be categorized into two classes: (1) automatic and (2) semiautomatic. The former class requires basically no user intervention in producing vessel centerlines. The later one needs at least a single user supplied point to kick off the execution. The automatic approach usually relies on a post-processing of the vascular segmentation. There were several methods worked along this line $[15,22,16,8]$. Nevertheless, if one wants to get a satisfactory centerline extraction, a topologically and morphologically correct segmention (with no handles and cavities) is compulsory which indeed is very difficult to obtain from clinical data. Automatic methods that detect centerlines directly from grayscale volumes based upon filtering method [11] and level set evaluation [14] were proposed. The performance of these algorithms depends on a set of global parameters that is not trivial to determine. Also, there is either zero local controllability of the solution or further post-processing is required to connect detached centerlines and expunge any plausible vessel axis.

Semiautomatic algorithms that require a single user supplied start point include mathematical morphology-based method [21] and iterative tracking (tracer-based) methods $[1,5,20]$. The tracer-based methods are capable of producing centerline with subvoxel accuracy. Vessel axis is traced from the user-defined point progressively. The direction of the next axial point is determined locally with a structural analysis. Florin et al. [5] exploited a Monte Carlo sampler to enable tracking of branches at furcation. In one of our previous works [20], we posed the tracking problem on a probabilistic framework to allow interactive user intervention in the middle of execution.

Tracking a centerline with a single start point, however, is inadequate in some applications in which the user wants 
to control the destination point of the tracking. To solve this problem, methods that permit users to supply start and end points were developed $[4,19,12]$. One of these methods exploit a minimal cost path algorithm on a graph defined by the voxel spatial dimensions [4]. Wink et al. [19] and $\mathrm{Li}$ and Yezzi [12] added one extra non-spatial dimension (scale/vessel thickness) to the graph to make tracking of the vessel interior more accurate. Nonetheless, the centerline extracted consists of a sequence of connected voxels, which is a very complex representation of vessel axis and indeed it has no subvoxel accuracy.

The closest work related to this paper is the work by Frangi et al. [6]. They modeled a vessel centerline with a parameteric curve (B-spline) and searched the solution by minimizing an energy functional defined along the curve. The number of spline control points (knots) has to be predefined. This makes their algorithm incapable of extracting centerline of complex vasculature if this number is small, or if there are too many knots redundant complexity is eventuated. During the evaluation of the objective function, numerous interpolations of an image volume are required. These operations are computationally expensive and may prone to interpolation artifacts. In addition, if the user wants to constrain the solution to pass through certain locations, insertion of anchor points is not trivial in their algorithm. This is because spline knots are not on the curve itself; thus implementation of anchors has to be coupled with the complexity of B-spline.

We propose a novel approach to attack the centerline extraction problem with a non-parameteric curve and a polygonal line algorithm that finds principal curve through an image volume. A vesselness measure is used to characterize vessel centerlines as in [6]. Besides, we utilize its corresponding scale-space data to estimate the local vessel width; and hence, vascular segmentation is obtained as a byproduct in our method.

All the aforementioned flaws in [6] are eliminated on this novel framework. Thanks to the polygonal line representation, arbitrary number of vertices can be added to the curve to make the centerline adaptive to vasculature complexity. Users have full controllability in our method; additional anchor points can be inserted, if needed, to handle difficult situation, such as diseased portions and kissing vessels. We treat voxel information as data points in a 3D space (point cloud) and find a curve that passes through the "middle" of points whose locations show higher chance of existence of tubular structures. Because of this, no interpolation is needed. Our method comprehends more informationdata points around the non-parameteric curve within its local width-in determining a centerline, contrary to sole dependency on the interpolated data along the spline as in [6]. Furthermore, in experiments with 3D synthetic image volumes, a magnetic resonance angiography (MRA) and a computerized tomography angiogram (CTA), we demonstrate that our centerline has subvoxel accuracy and high robustness to variation in image resolution, voxel anisotropy and image noise.

\section{Principal Curves to Extract Centerline and Estimate Local Width}

In this section, the theoretical background of principal curves in extracting tubular structure centerline in grayscale image volumes is discussed (Section 2.1). We then briefly present the algorithm in Section 2.2. Details of three core steps are given in Sections 2.3 and 2.4. Finally, a method to derive useful information for estimating local vessel width is presented (Section 2.5).

\subsection{Background}

Principal curves are a generalization of linear principal components [9]. Consider a $d$-dimensional random variable $\mathbf{X}=\left(X_{1}, \ldots, X_{d}\right)$ with finite second moments. The first linear principal component is a straight line passing through $\mathbb{X}$ (a set of realization of $\mathbf{X}$ ) which minimizes the average squared Euclidean distance to points $\mathbf{x} \in \mathbb{X}$. This line gives the best linear summary of the data. Hastie and Stuetzle [9] relaxed this linear requirement of principal components by defining the notion of principal curves. Later Kégl et al. [10] refined it by incorporating a length constraint.

According to their definition, a smooth curve $\mathbf{f}(t)=$ $\left(f_{1}(t), \ldots, f_{d}(t)\right)$ is a principal curve in $\mathbb{R}^{d}$ parameterized by a real argument $t$ if

1. $\mathbf{f}$ does not intersect itself, i.e., $a \neq b \Rightarrow \mathbf{f}(a) \neq \mathbf{f}(b)$,

2. f has a given length $L$ inside any bounded region in $\mathbb{R}^{d}$, and

3. $\mathbf{f}$ is self-consistent, i.e., $\mathbf{f}(t)=E\left(\mathbf{X} \mid \mathbf{f}^{-1}(\mathbf{X})=t\right)$, where $\mathbf{f}^{-1}(\mathbf{x})$ is known as the projection index of point $\mathbf{x}$ on the curve $f$

$$
\mathbf{f}^{-1}(\mathbf{x})=\sup _{t}\left\{t:\|\mathbf{x}-\mathbf{f}(t)\|=\inf _{\tau}\|\mathbf{x}-\mathbf{f}(\tau)\|\right\},
$$

the operator $\|\cdot\|$ denotes $l^{2}$-norm in $\mathbb{R}^{d}$. This projection index is the parametric variable $t$ whose location on the principal curve $f$ has the shortest orthogonal distance (denoted by inf $\|\cdot\|)$ to the point $\mathbf{x}$. If there exists more than one such parametric variable, the maximum one is chosen to be the projection index (denoted by $\sup \{\cdot\}$ ).

It is the self-consistency property of principal curves that attracts our attention. This property is similar to the equidistance property of medial axis of boundaries and shapes. Self-consistency means that every point on the principal curve coincides the "middle"/expected location of all the data points that are closest to itself. 
Along a similar research line $[2,10,13]$, we apply principal curves to extract tubular structures in grayscale image volumes, particularly their centerline. Nonetheless, it is very different from those previous works that we are working on non-binary data. Every input volume voxel center is regarded as a data point. The self-consistency is defined locally w.r.t. the width of a principal curve and by the voxel intensities. The locality criterion is to neglect the influence from irrelevant structures that are relatively far away from vessel of interest (e.g., adjacent objects of no interest and branches at furcation) in the image volume. The principal curve to determine the centerline of a tubular structure is therefore expressed as

$$
\begin{aligned}
\mathbf{f}(t) & =E\left(\mathbf{X} \mid \mathbf{f}^{-1}(\mathbf{X})=t\right) \\
& =\frac{\sum_{\mathbf{x} \in \mathbb{X}, \mathbf{f}^{-1}(\mathbf{x})=t} \mathbf{x} P(\mathbf{x})}{\sum_{\mathbf{x} \in \mathbb{X}, \mathbf{f}^{-1}(\mathbf{x})=t} P(\mathbf{x})},
\end{aligned}
$$

and the projection index is given by

$$
\begin{aligned}
\mathbf{f}^{-1}(\mathbf{x})=\sup _{t}\{t: & \|\mathbf{x}-\mathbf{f}(t)\|= \\
& \left.\min \left(r(t), \inf _{\tau}\|\mathbf{x}-\mathbf{f}(\tau)\|\right)\right\},
\end{aligned}
$$

where $r(t)$ is the associated local width of a tubular structure delineated by the principal curve $\mathbf{f}$ at $t$, data point $\mathbf{x}$ denotes a voxel center, and $P(\mathbf{x})$ gives the existence probability of a tubular structure centerline at $x$ after normalization with the denominator.

\subsection{The Overall Algorithm}

The overall algorithm to extract tubular structure centerline is summarized in a flow diagram, as shown in Figure 1. It is based on an efficient algorithm proposed by Kégl et al. [10] to find principal curves, namely polygonal line algorithm. The output is a set of polygonal lines with $k$ segments under a length constraint that minimizes the average squared distance over input data points (image voxels in our case). Hence, the output complexity is $O(k)$, which is typically much less than the number of image voxels. A typical image volume usually consists of millions of voxels. Nonetheless, less than a hundred points is normally sufficient to model the centerline of a tubular structure which is supposed to be smooth. This makes the polygonal line algorithm an appropriate method to extract vessel centerlines, contrary to the other two algorithms $[9,18]$ that give outputs with high complexity (number of curve points equals the number of image voxels).

The algorithm begins with a user-defined curve. This curve depicts a segment of tubular structure of interest. It consists of at least two end vertices. The user picks the vertices on planar reformatted images of an image volume. The two end vertices have to be selected at the centerline of

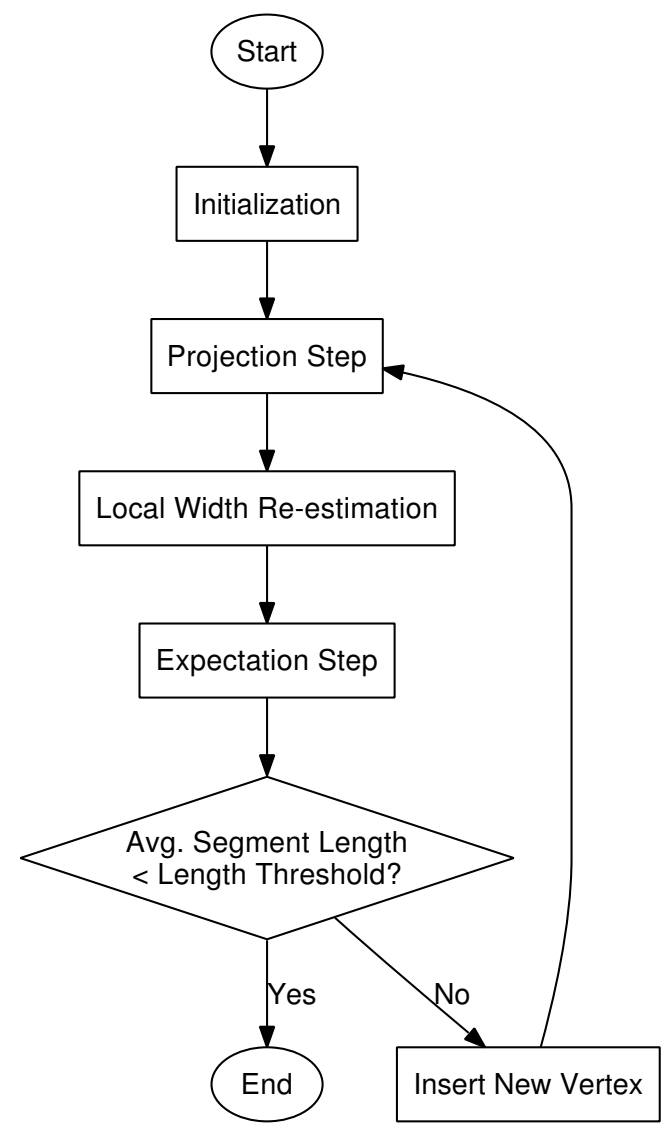

Figure 1. Flow diagram of the polygonal line algorithm to extract tubular structure centerline.

the structure of interest. Those two vertices are fixed (not optimized) to avoid undesired shortening/lengthening of the estimated centerline. This permits the user to gain a certain control over the algorithmic output. If accurate locations of the two end vertices is difficult to identify from the reformatted images, plausible position can be computed, as suggested in [11], via finding the zero crossing of two functions defined by image gradients and eigenvectors of Hessian matrices (which denote the basis of a vessel cross-section) in a local neighborhood. Additional vertices may be required if the structure of interest deviates from a straight line significantly. Precise locating of additional vertices is, however, not necessary (unless they are anchors, locations where the centerline must pass through). Local widths of the initial curve components (vertices and segments) are set to an arbitrary value slightly greater than the physical size of an image voxel.

Following the initialization is the projection step and reestimation of local width (Section 2.3). In the expectation step, movable vertices (anchors are excluded as they are fixed) are optimized sequentially with an objective function (Section 2.4). This objective function encourages the 
curve to pass through the "middle" of a tubular structure. A suboptimal solution is obtained with a gradient descent method. Stopping criteria of the optimizer can be defined by the maximum number of iterations, a tolerance on the gradient magnitude or changes in optimization parameters (i.e., coordinates of polygonal line vertex). Once the optimizer terminates, a new vertex is inserted to the longest segment. This is to make the vertex sampling as even as possbile. Then the algorithm goes to the next iteration. It keeps iterating until the average curve segment length is below a length threshold defined as a scalar multiple of the voxel physical size. This is a straightforward stopping criterion of the polygonal line algorithm for tubular structure centerline extraction.

\subsection{Projection Step and Local Width Estimation}

In theory, the goal in the projection step is to determine the projection index $\mathbf{f}^{-1}(\mathbf{x})$ of every data point $\mathbf{x}$ given the current principal curve f. However, knowing the exact index is not necessary, as we are not going to update the principal curve on the parametric space. One elegant aspect of the polygonal line algorithm is that the representation of principal curve is very simple. It consists of only $k+1$ vertices $\mathbf{v}_{1}, \ldots, \mathbf{v}_{k+1}$ and $k$ line segments $\mathbf{s}_{1}, \ldots, \mathbf{s}_{k}$. Therefore, it is sufficient to determine which component (either a vertex or a segment) does a data point project onto.

We define $2 k+1$ disjoint sets $\mathbb{V}_{1}, \ldots, \mathbb{V}_{k+1}, \mathbb{S}_{1}, \ldots, \mathbb{S}_{k}$ to hold the data points that projected onto vertices and segments of $\mathbf{f}$, respectively,

$$
\begin{gathered}
\mathbb{V}_{i}=\left\{\mathbf{x} \in \mathbb{X}: \mathbf{x} \notin \bigcup_{j<i} \mathbb{V}_{j}, \delta_{v}\left(\mathbf{x}, \mathbf{v}_{i}\right) \leq \frac{W\left(\mathbf{v}_{i}\right)}{2},\right. \\
\left.\delta_{v}\left(\mathbf{x}, \mathbf{v}_{i}\right)=\min \left(\inf _{m} \delta_{v}\left(\mathbf{x}, \mathbf{v}_{m}\right), \inf _{p} \delta_{s}\left(\mathbf{x}, \mathbf{s}_{p}\right)\right)\right\},
\end{gathered}
$$

where $\delta_{v}\left(\mathbf{x}, \mathbf{v}_{i}\right)=\left\|\mathbf{x}-\mathbf{v}_{i}\right\|, \delta_{s}\left(\mathbf{x}, \mathbf{s}_{i}\right)$ returns the shortest Euclidean distance from point $\mathbf{x}$ to the segment $\mathbf{s}_{i}$ and $W\left(\mathbf{v}_{i}\right)$ is the local width associated with the vertex $\mathbf{v}_{i}$,

$$
\begin{aligned}
& \mathbb{S}_{i}=\left\{\mathbf{x} \in \mathbb{X}: \mathbf{x} \notin \bigcup_{\forall m} \mathbb{V}_{m}, \mathbf{x} \notin \bigcup_{p<i} \mathbb{S}_{p},\right. \\
& \left.\delta_{s}\left(\mathbf{x}, \mathbf{s}_{i}\right) \leq \frac{W\left(\mathbf{s}_{i}\right)}{2}, \delta_{s}\left(\mathbf{x}, \mathbf{s}_{i}\right)=\inf _{j} \delta_{s}\left(\mathbf{x}, \mathbf{s}_{j}\right)\right\},
\end{aligned}
$$

where $W\left(\mathbf{s}_{i}\right)$ is the local width associated with the segment $\mathbf{s}_{i}$.

In other words, in the projection step, every data point is placed into either a projection set of vertex $\mathbf{v}$ or segment $\mathbf{s}$ if it is laid within the territory defined by the component's local width $W(\cdot)$ (analogous to the diameter of the tube to be extracted). All the points in a particular projection set share the same property that they are the closest to the component but nothing else.
Suppose for each data point $\mathbf{x}$ we have two scalar values, $\varepsilon(\mathbf{x})$ and $\omega(\mathbf{x})$, associated with it. $\varepsilon(\mathbf{x})$ represents likeliness of the existence of tubular structure and $\omega(\mathbf{x})$ denotes the scale at which the likeliness is computed. Local width of the principal curve can be derived from these two quantities. $\varepsilon(\cdot)$ gives the confidence level of the scale-space data $\omega(\cdot)$ which is proportional to the width of a nearby tubular structure. Hence, local width of the $i^{\text {th }}$ principal curve segment $W\left(\mathbf{s}_{i}\right)$ can be calculated as the expected scale-space data of points in the projection set $\mathbb{S}_{i}$. Mathematically, it is given by

$$
W\left(\mathbf{s}_{i}\right)=\kappa \frac{\sum_{\mathbf{x} \in \mathbb{S}_{i}} \omega(\mathbf{x}) P_{i}(\mathbf{x})}{\sum_{\mathbf{x} \in \mathbb{S}_{i}} P_{i}(\mathbf{x})},
$$

where $\kappa$ is a constant that transfers the expected scale-space data to the width of the detected tubular structure (see Section 2.5), the denominator is for normalization and

$$
P_{i}(\mathbf{x})=\exp \left(-\frac{\left(1-\varepsilon(\mathbf{x}) / \max _{\mathbf{y} \in \mathbb{S}_{i}} \varepsilon(\mathbf{y})\right)^{2}}{2(1-C)^{2}}\right),
$$

denotes an existence probability of a tubular structure at data point $\mathbf{x}$. $C$ is a cutoff value of the normalized likeliness in the exponent numerator, which controls the sensitivity of $P_{i}(\cdot)$ towards $\varepsilon(\cdot)$. The cutoff value can be either found empirically or calibrated with synthetic data with known tubular width. Regarding the local width associated with vertices of the principal curve, we set $W(\mathbf{v})$ to the maximum local width of its adjacent segment(s). A method to compute $\varepsilon(\cdot)$ and $\omega(\cdot)$ from grayscale image volumes is described in Section 2.5.

\subsection{Objective Function in Expectation Step}

The objective function in the expectation step consists of three cost functions. The first one implements the selfconsistency criterion and the other two cost functions are for regularization. The implementation of Equation 2 can be achieved by minimizing the expected normalized Euclidean distance from projected data points to every vertex $\mathbf{v}_{i}$ and its adjacent segments. This encourages the polygonal lines to pass through the "middle" of regions where tubular structure existence probability is high. The cost function is given as

$$
\begin{gathered}
\underbrace{}_{\text {data }}(\underbrace{\sum_{j \in \mathcal{N}\left(\mathbf{v}_{i}\right)} \sum_{\mathbf{x} \in \mathbb{S}_{j}} \frac{\delta_{s}\left(\mathbf{x}, \mathbf{s}_{j}\right)}{W\left(\mathbf{s}_{j}\right) / 2} P_{j}(\mathbf{x}) / \sum_{j \in \mathcal{N}\left(\mathbf{v}_{i}\right)} \sum_{\mathbf{x} \in \mathbb{S}_{j}} P_{j}(\mathbf{x})}_{\text {Adjacent segment( })}) \\
+(\underbrace{\sum_{\mathbf{x} \in \mathbb{V}_{i}} \frac{\delta_{v}\left(\mathbf{x}, \mathbf{v}_{i}\right)}{W\left(\mathbf{v}_{i}\right) / 2} P_{i}^{\prime}(\mathbf{x}) / \sum_{\mathbf{x} \in \mathbb{V}_{i}} P_{i}^{\prime}(\mathbf{x})}_{\text {Vertex (neglected) }})
\end{gathered}
$$


where $\mathcal{N}\left(\mathbf{v}_{i}\right)$ denotes a set of segment indices whose segment is adjacent to the vertex $\mathbf{v}_{i}, P_{j}(\mathbf{x})$ is the function given in Equation 7 for measuring the existence probability of tubular structure and $P_{i}^{\prime}(\mathbf{x})$ is a similar function but with the segment projection set $\mathbb{S}$ replaced by a vertex projection set $\mathbb{V}$. The function $\delta .(\cdot)$ returns the shortest Euclidean distance from a data point to a principal curve component (defined in Section 2.3). It is normalized by the local half width of the component $\frac{W(\cdot)}{2}$. Since $\delta .(\cdot) \leq \frac{W(\cdot)}{2}$ (cf. it is one of the conditions in the set definitions given in Equations 4 and 5), and their weights $P_{j}(\cdot)$ and $P_{i}^{\prime}(\cdot)$ are normalized by the denominator, value of $E_{\text {data }}(\cdot)$ is in the interval $[0,1]$.

In practice, the contribution from vertex in $E_{\text {data }}\left(\mathbf{v}_{i}\right)$ tends to drive the vertex $\mathbf{v}_{i}$ towards the outer curvature region (area opposite to the curvature center). This is because the projection region of vertex is squeezed into a plane in the inner curvature area, and thus very likely there is no data point projected onto the vertex. This asymmetric distribution of data points in $\mathbb{V}_{i}$ induces bias towards the outer curvature region as the expected distances to those data points are minimized. To get rid of this bias, we neglect the second addend in Equation 8.

The second cost function accounts for the relative strain induced by vertex dislocation due to optimization. Strain is a measure on the degree of deformation. It is expressed as the difference in placement of parts in a system. Numerically, the relative strain is given as

$$
E_{\text {strain }}\left(\mathbf{v}_{i}\right)=\frac{\sum_{j \in \mathcal{N}\left(\mathbf{v}_{i}\right)} l\left(\mathbf{s}_{j}\right)-\sum_{j \in \mathcal{N}\left(\mathbf{v}_{i}\right)} l\left(\mathbf{s}_{j}^{0}\right)}{\sum_{j \in \mathcal{N}\left(\mathbf{v}_{i}\right)} l\left(\mathbf{s}_{j}^{0}\right)},
$$

where $l(\cdot)$ gives the length of the argument segment, $\mathbf{s}_{j}$ and $\mathbf{s}_{j}^{0}$ denote the adjacent segments after and before the vertex optimization, respectively. $E_{\text {strain }}(\cdot)$ returns value in $\mathbb{R}$. It calculates the relative change in segment length due to the optimization, e.g. 0 denotes no change, 1 dentoes $100 \%$ lengthening and -0.2 means $20 \%$ shortening.

The last cost function penalizes unevenness of vertices in the polygonal lines. Even sampling of vertices in the principal curve is essential to estimating a smooth tubular structure centerline. Too few/many vertices in a region would result in a straight lengthy curve/redundancy. This cost function measures the length ratio of adjacent segments at vertex $\mathbf{v}_{i}$. It is expressed as

$$
E_{\text {evenness }}\left(\mathbf{v}_{i}\right)=1-\frac{\min \left\{l\left(\mathbf{s}_{j}\right): j \in \mathcal{N}\left(\mathbf{v}_{i}\right)\right\}}{\max \left\{l\left(\mathbf{s}_{j}\right): j \in \mathcal{N}\left(\mathbf{v}_{i}\right)\right\}},
$$

giving a value in range $[0,1] ; 0$ denotes equal length in adjacent segments indicating even vertex spacing in a local neighborhood, if a segment is much longer than the other one $E_{\text {evenness }}(\cdot) \rightarrow 1$.

In summary, the objective function exploited in the ex- pectation step is given as,

$$
\begin{aligned}
E\left(\mathbf{v}_{i}\right)=E_{\text {data }} & \left(\mathbf{v}_{i}\right)+ \\
& \lambda \frac{k}{k^{0}}\left(E_{\text {strain }}\left(\mathbf{v}_{i}\right)+E_{\text {evenness }}\left(\mathbf{v}_{i}\right)\right),
\end{aligned}
$$

where $\lambda \in \mathbb{R}^{+}$is a variable to control the influence of the regularization terms. $k$ and $k^{0}$ are the numbers of vertices in the current and initial curves, respectively. The multiplication of their ratio is to increase the importance of regularization as the number of vertices increases to avoid overfitting.

\subsection{Vesselness Measure and Scale-space Data}

The tubular structure existence likeliness $\varepsilon(\cdot)$ and the scale-space data $\omega(\cdot)$ exploited in Section 2.3 are computed with the multiscale line filter proposed by Sato et al. [17]. This method performs multiscale analysis on image volume with the Hessian matrix to enhance bright tubular structures. Filter responses are calculated based upon the eigenvalues of Hessian matrix. Scale is referred to the $\sigma$ parameter of the second derivatives of Gaussian used in the Hessian matrix computation. Multiple responses at discrete scales are computed and aggregated with a maximum operator. We use the aggregated responses as $\varepsilon(\cdot)$ in this work. Higher response means higher likeliness of an existence of tubular structure. The scale that has been chosen by the maximum operator is kept in $\omega(\cdot)$ as a scale-space datum. It provides us with information on the local width of an enhanced tubular structure. Sato et al. [17] found experimentally that, for a tube with bar-shaped cross-sectional profile, if $\sigma$ equals half of the tube width, maximum vesselness measure is observed along the centerline in the scale-space. This suggests that $\sigma$ is an estimate of the half tube width and thus we have $\kappa=2$ in Equation 6.

\section{Experimental Results}

We have designed two synthetic data sets to demonstrate the robustness of the proposed method to noise, changes in image resolution and voxel anisotropy. They are both in matrix size $200 \times 60 \times 60$ with voxel size $1 \times 1 \times 1$ arbitrary unit (a.u.), one of the sets is an image of a straight tube with varying width $(2 \times$ its radius) and the other set imaged a curved tube in a fixed width. The straight tube runs along the $\mathrm{X}$-axis in the middle of the image volume. It starts with a local width 16 a.u., changes progressively to 8 a.u. between the $40^{\text {th }}-80^{\text {th }}$ a.u. from the start point, and increases to 12 a.u. between the $120^{\text {th }}-160^{\text {th }}$ a.u. from its origin. Figures 2(a)-(b) show the image volume and the tube. Voxels inside the tube have intensity equals 255 , for those outside, they are set to 0 . This synthetic data set is to evaluate our method in estimating the local width of a tubular structure. The curved tube given in Figure 2(d) runs along the $\mathrm{X}$-axis, 


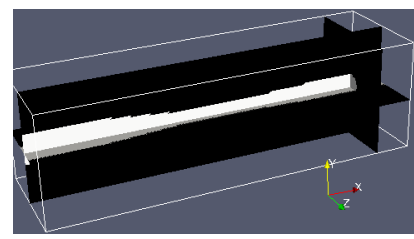

(a)

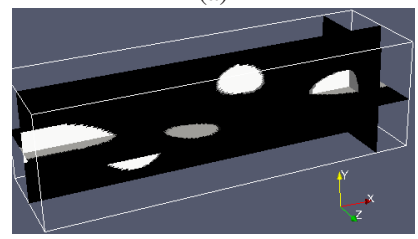

(c)

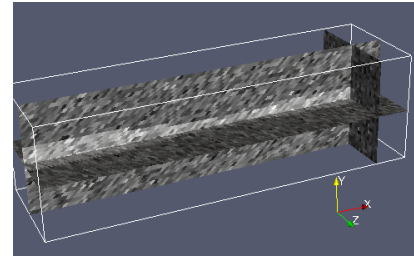

(e)

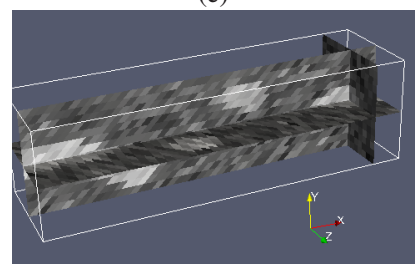

(g)

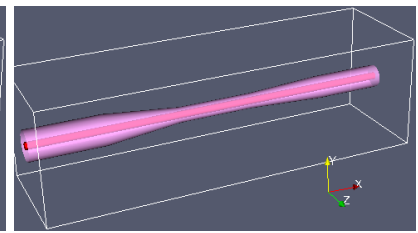

(b)

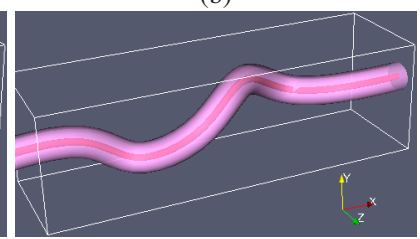

(d)

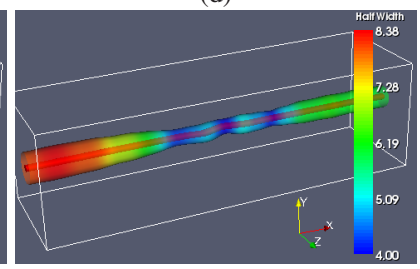

(f)

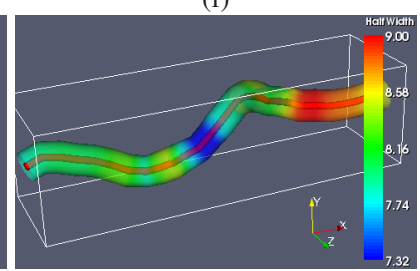

(h)
Figure 2. (a)-(d) Synthetic data sets and the generating models (both centerlines and tube surfaces). Slices from noiseless straight tube data set (a) and its model (b). Slices from noiseless curved tube data set (c) and its model $(d)$. (e)-(h) Their noisy counterparts, extracted centerlines and tubes built upon estimated local widths. Slices from the $5 \mathrm{~dB}$ (downsampled by a factor of 2) straight tube (e) and the $10 \mathrm{~dB}$ (downsampled by a factor of 4) curved tube $(\mathrm{g})$. Centerlines and tube models estimated from noisy straight $(f)$ and curved $(h)$ tubes. Surfaces are color-coded with the half local width (in a.u.).

starts rotating around the axis anti-clockwisely for $360^{\circ}$ at the $25^{\text {th }}$ a.u. from its origin and goes back to the $\mathrm{X}$-axis at the $175^{\text {th }}$ a.u. from the start point. Its local width is kept at 16 a.u., voxel intensity 255 denotes interior part and 0 represents the exterior. We evaluate the locations of the extracted tube centerline with this data set at various image resolutions and noise conditions.

The experiments are conducted at the following conditions: isotropic downsampling by factors of 1,2 and 4 for the straight tube and 1, 4 and 8 for the curved tube; anisotropic downsampling in the Z-direction by factors of 4 and 8 for the straight and curved tube, respectively; and adding zero-mean Gaussian noise at signal-to-noise (SNR) 10 and $5 \mathrm{~dB}$. These are to assess the effects of changes in image resolution, voxel anisotropy and noise level on the algorithmic performance, respectively. Downsampling is performed with a linear interpolation method. SNR is defined

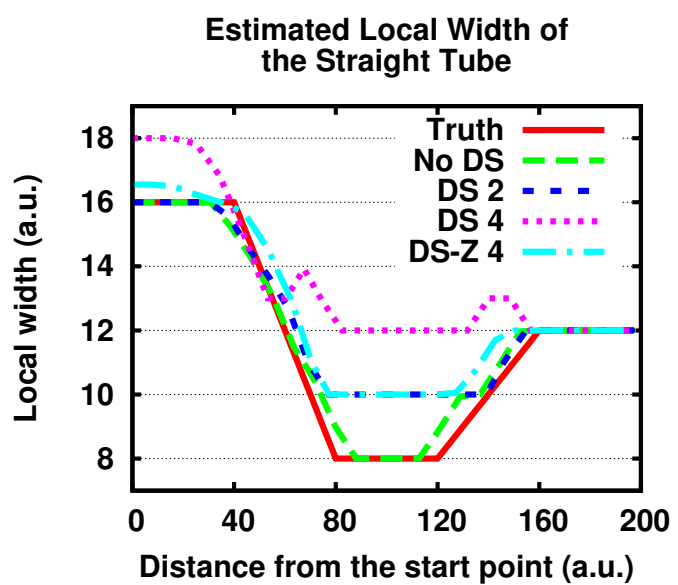

(a)

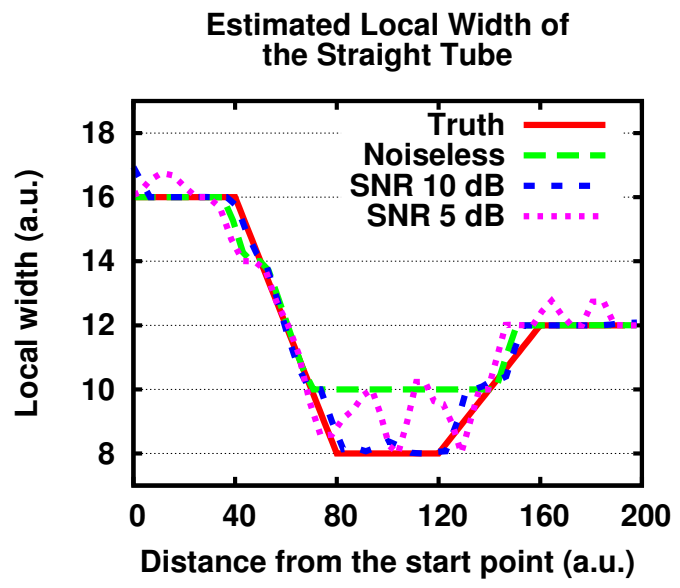

(b)

Figure 3. Local width estimated along the centerline extracted vs. the truth at different downsampling conditions (a) and noise levels (b). DS denotes downsampling by the factor followed, DS-Z denotes downsampling in Z-direction.

as $20 \log _{10}\left(A / \sigma_{g}\right)$, where $A=255$ is the maximum intensity of the image and $\sigma_{g}$ is the standard deviation of the additive Gaussian noise. $\mathrm{SNR}=10 \mathrm{~dB} \Rightarrow \sigma_{g}=80.6$ and $\mathrm{SNR}=5 \mathrm{~dB} \Rightarrow \sigma_{g}=143.4$. Image slices from a $5 \mathrm{~dB}$ straight tube and a $10 \mathrm{~dB}$ curved tube are given in Figures 2(e) and 2(g), respectively. Once noise is added, intensities are renormalized back to their original range (i.e., $0-255$ ). Local width estimations are evaluated visually with line plots (local width vs. running distance from the start point) against the truth local width. Accuracy of the estimated centerline locations is quantified with the shortest Euclidean distance to the truth.

Experimental results are presented in Figure 3 and Table 1. Figure 3(a) shows the local width estimated at different downsampling conditions. Seven discrete scales are exploited in the computation of the vesselness measure covering $3-9$ a.u., they are linearly sampled. The cutoff vari- 
able $C$ in Equation 7 and the weight $\lambda$ of the regularization terms in Equation 11 are determined empirically with the noiseless straight and curved tube digital phantom, respectively. It is found that $C=0.975$ and $\lambda=0.2$ generally produce good width and centerline estimation. The stopping criterion of the algorithm, average length of the polygonal line segments, is set to 2 voxels of the lowest resolution (i.e., $2 \times 8=16$ a.u.). At the original resolution, width estimation is accurate, although there are small discrepancies in the transition regions $\left(40^{\text {th }}-80^{\text {th }}\right.$ and $120^{\text {th }}-160^{\text {th }}$ a.u. from the start point). Those discrepancies probably are due to the discrete sampling in the scale-space. Errors are observable at the narrow portion of the tube when the image volume is downsampled by a factor of 2 isotropically and anisotropically along the Z-direction by 4 . Such errors are prone to the resolution limit; if there is 1 voxel error in the estimation, the local widths approximated are 10 and 12 a.u. (truth is 8 a.u.) for those two downsampling conditions, respectively. Errors are more significant in the volume downsampled by a factor of 4 . But still they are less than or equal to the worst case (12 a.u.) owing to the resolution constraint.

For the noise sensitivity experiments, we apply the algorithm to an image volume with resolution equals $1 / 2$ of the original. Results are presented in Figure 3(b). The error due to the limitation of image resolution is alleviated in a noisy condition (local widths estimated are more accurate, less than 10 a.u., in general at the narrowed region in noisy data than the noiseless counterpart, indeed a noiseless image is very rare in a clinical environment). Large error with the noiseless data may owe to intensity bias induced by image averaging. This bias makes the narrowed region apparently wider. Random noise and intensity rescaling may curtail such biased effect eventuating in a closer width estimation. Even in a severe noise condition (SNR $=5 \mathrm{~dB}$ ), we found that the estimation is satisfactory; the approximation errors are within the error level due to the resolution limit. Figure 2(f) shows the centerline estimated and a tube model constructed with the approximated local width (surface is colorcoded with the half local width) of the synthetic straight tube data set at noise level $5 \mathrm{~dB}$.

Percentile statistics of the shortest Euclidean distance (in a.u.) to the truth centerline locations of the curved tube are tabulated in Table 1. The results are obtained with the stopping criterion of the algorithm set to 2 voxels of the image resolution. Image resolutions are listed in the second column of Table 1. 50\%, $75 \%$ and $100 \%$ percentiles of the data are given. The $100 \%$ percentile figures correspond to the maximum shortest Euclidean distance to the truth. In the brackets, distances are expressed as a fraction of a single voxel, a value $<1$ (in bold face) means the distance is shorter than the physical size of a voxel. The results show subvoxel accuracy in all situations, even in the $100 \%$ percentile. Exceptions are observed at the original resolution.
Table 1. Percentile statistics of the shortest Euclidean distance (in a.u.) to the truth centerline locations of the curved tube. DS denotes downsampling by the factor followed, $D S-Z$ denotes downsampling in Z-direction, $\infty d B$ means noiseless data set. Figures in brackets are ratios of the distances to the voxel size, a value $<1$ is highlighted in bold face emphasizing subvoxel precision.

\begin{tabular}{c|c|ccc} 
& $\begin{array}{c}\text { Voxel } \\
\text { size }\end{array}$ & \multicolumn{3}{|c}{ Percentiles of the shortest Euclidean } \\
& distance to the truth locations (a.u.) \\
& a.u.) & $50 \%$ & $75 \%$ & $100 \%$ \\
\hline DS 1 & 1 & $1.28(1.28)$ & $1.80(1.80)$ & $3.25(3.25)$ \\
DS 4 & 4 & $1.16(\mathbf{0 . 2 9})$ & $1.93(\mathbf{0 . 4 8})$ & $3.02(\mathbf{0 . 7 5})$ \\
DS 8 & 8 & $2.84(\mathbf{0 . 3 6})$ & $3.47(\mathbf{0 . 4 3})$ & $4.75(\mathbf{0 . 5 9})$ \\
DS-Z 8 & 8 & $1.43(\mathbf{0 . 1 8})$ & $2.23(\mathbf{0 . 2 8})$ & $3.63(\mathbf{0 . 4 5})$ \\
\hline \hline$\infty \mathrm{dB}$ & 4 & $1.16(\mathbf{0 . 2 9})$ & $1.93(\mathbf{0 . 4 8})$ & $3.02(\mathbf{0 . 7 5})$ \\
$10 \mathrm{~dB}$ & 4 & $1.15(\mathbf{0 . 2 9})$ & $1.86(\mathbf{0 . 4 7})$ & $3.16(\mathbf{0 . 7 9})$ \\
$5 \mathrm{~dB}$ & 4 & $1.24(\mathbf{0 . 3 1})$ & $2.16(\mathbf{0 . 5 4})$ & $3.54(\mathbf{0 . 8 8})$ \\
\hline
\end{tabular}

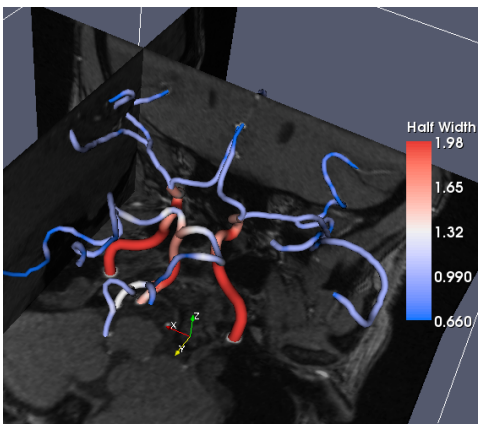

(a)

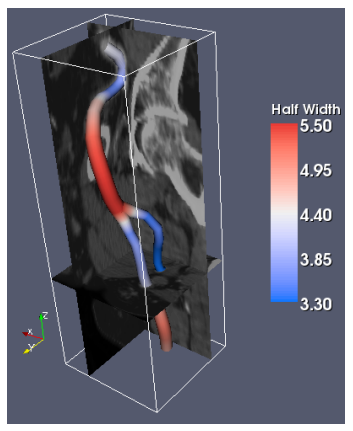

(b)
Figure 4. Slices from two clinical data sets, time-of-fight MRA of a cerebral circulation (a) and CT of a left pelvis showing the iliac arteries (b), overlaid with the major vessels extracted. Tube surfaces are color-coded with the half local width (in $\mathrm{mm}$ ).

We found that it is due to the shifting of the maximum vesselness measure towards the center of curvature. Indeed it is evidenced that our algorithm successfully tracks the location where the vesselness measure is locally maximum.

All these experimental results suggest that the proposed method is capable of determining tube centerlines in image volumes with subvoxel accuracy. They also show that the centerlines extracted have high robustness to noise, changes in image resolution and voxel anisotropy. Furthermore, our algorithm can produce accurate estimation of tubular structure local width (subjected to the resolution limit).

To demonstrate the application to clinical data, we have tested our algorithm on two publicly available ${ }^{1}$ data sets, they are a time-of-flight MRA of a cerebral circulation in isotropic voxels and a CT of a left pelvis imaged iliac arteries in anisotropic voxels. Figure 4 shows the major vessels extracted. In our experience, $3-5$ supplementary vertices (in addition to two fixed end vertices) chosen nearby the desired centerline is sufficient to give a good initialization to

\footnotetext{
${ }^{1}$ Available at http://www.gris.uni-tuebingen.de/edu/ areas/scivis/volren/datasets/new.html.
} 
extract a long vessel segment.

\section{Conclusion}

A novel approach to extracting centerline of vessels in a 3D angiography is proposed. Local vessel widths are approximated simultaneously while the centerline is extracted. The method is based upon an algorithm known as polygonal line algorithm. It is developed to find principal curve in a cloud of $d$-dimensional data points. By definition, a principal curve is a smooth curve that passes through the "middle" of all the data points that are closest to itself. It is a nonlinear generalization of principal component. On this framework, centerline is modeled as a set of vertices and a series of line segments that join the adjacent vertices. Local width information is stored in each component (vertex or segment) of the curve. As such vascular segmentation can be obtained as a byproduct.

We have discussed, from a theoretical view point, on how principal curve can be defined for extraction of tubular structures, and proposed a novel algorithm for this application. Our algorithm takes an iterative approach. It consists of a projection step, re-estimation of local width of the polygonal lines, and an expectation step. No data interpolation is needed in this method. Centerlines extracted are adaptive to the vasculature complexity; arbitrary number of vertices can be added to the curve in order to model a long vascular segment. Additional anchor points (that the centerline must pass through) can also be inserted to handle difficult centerline extraction situation. We have evaluated the method on two synthetic data sets. Experimental results show that centerlines extracted are in subvoxel accuracy and local widths estimated are within the error level due to the limit of image resolution. Furthermore, it is demonstrated that our new method has high robustness to noise, variation in image resolution and voxel anisotropy. We have also applied the proposed method to two publicly available clinical data sets, from which vessels with branches and of various width are extracted accordingly.

A more rigorous validation with relatively realistic synthetic data or physical phantom data is of interest for future research. In addition, it is worth studying the extraction of small-radius vessel segments in a noisy environment and comparing the performance of the proposed method with some close works.

\section{Acknowledgments}

The authors would like to acknowledge the support from the K. S. Lo Foundation.

\section{References}

[1] S. R. Aylward and E. Bullitt. Initialization, noise, singularities, and scale in height ridge traversal for tubular object centerline extraction.
IEEE Trans. Med. Imag., 21(2):61-75, 2002.

[2] J. D. Banfield and A. E. Raftery. Ice floe identification in satellite images using mathematical morphology and clustering about principal curves. J. Am. Stat. Assoc., 87(417):7-16, Mar. 1992.

[3] E. Bullitt, K. E. Muller, I. Jung, W. Lin, and S. Aylward. Analyzing attributes of vessel populations. Med. Imag. Anal., 9:39-49, 2004.

[4] T. Deschamps and L. D. Cohen. Fast extraction of minimal paths in 3D images and applications to virtual endoscopy. Med. Imag. Anal., 5(4):281-299, 2001.

[5] C. Florin, N. Paragios, and J. Williams. Particle filters, a quasi-monte carlo solution for segmentation of coronaries. In Med. Imag. Comput. Comput. Assist. Interv., pages 246-253, 2005.

[6] A. F. Frangi, W. J. Niessen, R. M. Hoogeveen, T. V. Walsum, and M. A. Viergever. Model-based quantitation of 3-D magnetic resonance angiographic images. IEEE Trans. Med. Imag., 18(10):946956, 1999.

[7] M. Groher, F. Bender, R.-T. Hoffmann, and N. Navab. Segmentationdriven 2D-3D registration for abdominal catheter interventions. In Med. Imag. Comput. Comput. Assist. Interv., pages 527-535, 2007.

[8] M. S. Hassouna, A. A. Farag, and R. Falk. Differential fly-throughs (DFT): a general framework for computing flight paths. In Med. Imag. Comput. Comput. Assist. Interv., number 3749 in Lecture Notes in Comput. Sci., pages 654-661. Berlin, Springer-Verlag, 2005.

[9] T. Hastie and W. Stuetzle. Principal curves. J. Am. Stat. Assoc., 84:502-516, 1989.

[10] B. Kégl, A. Krzyzak, T. Linder, and K. Zeger. Learning and design of principal curves. IEEE Trans. Pattern Anal. Machine Intell., 22(3):281-297, Mar. 2000.

[11] K. Krissian, C.-F. Westin, and R. Kikinis. Fast and automatic vessel centerline detection for MRA. In Int. Soc. Magn. Reson. Med., 2002.

[12] H. Li and A. Yezzi. Vessels as 4D curves: Global minimal 4D paths to extract 3D tubular surfaces. In A. Yezzi, editor, Comput. Vis. and Pattern Recogn., pages 82-82, 2006.

[13] X. Liu and Y. Jia. A bottom-up algorithm for finding principal curves with applications to image skeletonization. Pattern Recogn., 38:1079-1085, 2005.

[14] R. Manniesing, B. Velthuis, M. van Leeuwen, I. van der Schaaf, P. van Laar, and W. Niessen. Level set based cerebral vasculature segmentation and diameter quantification in CT angiography. Med. Imag. Anal., 10(2):200-214, Apr. 2006.

[15] R. L. Ogniewicz and O. Kubler. Hierarchy Voronoi skeletons. Pattern Recogn., 28(3):343-359, 1995.

[16] K. Palágyi, E. Sorantin, E. Balogh, A. Kuba, C. Halmai, B. Erdöhelyi, and K. Hausegger. A sequential 3D thinning algorithm and its medical applications. In Int. Conf. Info. Process. Med. Imag., volume 2082 of Lecture Notes in Comput. Sci., pages 409415. Springer-Verlag, 2001.

[17] Y. Sato, S. Nakajima, N. Shiraga, H. Atsumi, S. Yoshida, T. Koller, G. Gerig, , and R. Kikinis. 3D multi-scale line filter for segmentation and visualization of curvilinear structures in medical images. Med. Imag. Anal., 2(2):143-168, 1998.

[18] R. Tibshirani. Principal curves revisited. Stat. Comput., 2:183-190, 1992.

[19] O. Wink, W. J. Niessen, and M. A. Viergever. Multiscale vessel tracking. IEEE Trans. Med. Imag., 23(1):130-133, 2004.

[20] W. C. K. Wong and A. C. S. Chung. Probabilistic vessel axis tracing and its application to vessel segmentation with stream surfaces and minimum cost paths. Med. Imag. Anal., 11(6):567-587, Dec. 2007.

[21] P. J. Yim, P. L. Choyke, and R. M. Summers. Gray-scale skeletonization of small vessels in magnetic resonance angiography. IEEE Trans. Med. Imag., 19(6):568-576, 2000.

[22] Y. Zhou and A. W. Toga. Efficient skeletonization of volumetric objects. IEEE Trans. Visual. Comput. Graphics, 5(3):196-209, 1999. 